\title{
Colonización temprana de hongos micorrícicos arbusculares en trigo con aplicación de productos naturales en un Andisol de La Araucanía
}

\author{
Effect on early colonization of arbuscular mycorrhizal fungi in wheat with \\ application of natural products in Andisol of Araucanía Region
}

\author{
Claudia Castillo $^{1,2 *}$, Daniza Morales ${ }^{1}$, Francisco Fuentealba ${ }^{1}$, Víctor San Martín ${ }^{1}$
}

\begin{abstract}
RESUMEN
En un suelo serie Vilcún de la Región de La Araucanía se estableció un ensayo de rotación trigo (Triticum aestivum L.) variedad Crac-Baer usando como precultivo raps (Brassica napus L.). El objetivo del estudio fue determinar en condiciones de campo, el efecto de la aplicación de tres productos naturales (PN): Fosfobio (bacterias solubilizadoras de fosfatos); Myconate (formononetina) y Natural Green (carbonato de calcio) a las semillas del cereal. A los 44 días después de la siembra (DDS), 63 DDS y 77 DDS se evaluaron la micorrización temprana en las raíces de las plantas cuantificando los puntos de entrada (PE) del hongo, frecuencia e intensidad micorrícica y biomasa de raíz colonizada (BRC) junto con parámetros de la planta. El diseño correspondió a un cuadrado latino con cuatro tratamientos y cuatro repeticiones, con unidades experimentales de $12 \mathrm{~m}^{2}$. A los 44 DDS, con la aplicación de Myconate aumentaron los PE y frecuencia de colonización, mientras a los 63 DDS y 77 DDS, aumentó la intensidad micorrícica con la aplicación de Natural Green y Fosfobio, respectivamente. Este PN también a los 77 DDS, incrementó en forma significativa la BRC, sobre los otros PN. De los resultados, se concluye que existe un efecto beneficioso de la micorrización HMA en trigo por la aplicación de estos PN. Finalmente, estos estudios resultan pioneros en el tema de la sustentabilidad de los agroecosistemas, ayudando a dilucidar las relaciones que se establecen entre los microorganismos benéficos de la rizósfera.
\end{abstract}

Palabras clave: porcentaje de frecuencia micorrícica, intensidad micorrícica, biomasa de raíz colonizada.

\begin{abstract}
In a Vilcún series soil of La Araucanía Region was established a crop rotation wheat (Triticum aestivum L.) variety Crac-Baer using raps as preculture (Brassica napus L.).The aim of the study was to determine under field conditions, the effect on the application of three natural products (NP): Fosfobio (phosphate solubilizing bacteria); Myconate (formononetin) and Natural Green (calcium carbonate). At 44 days after sowing (DAS), 63 DAS and 77 DAS, plant parameters and early mycorrhizal parameters as entry points $(E P)$, frequency $(\% F)$, intensity $(\% M)$, and colonized root biomass $(B R C)$ were evaluated. The experimental design was a Latin square with four replicates per treatment corresponded to 16 units of $12 \mathrm{~m}^{2}$. At 44 DAS, the results show that early colonization with application of Myconate, the EP and \%F increased compared to control. With the application of PN, it increased mycorrhizal intensity, at 63 DAS increase with the application of Natural Green, whilst at 77 DAS, was highest with Fosfobio, compared to control. Also, this PN, at 77 DAS, significantly increased the BRC, over the rest of PN. From the results, it is concluded that there is a beneficial effect of mycorrhization HMA in wheat due to the application of these PN. Finally, these studies are pioneers in the field of sustainability in agroecosystems helping to elucidate the relationships established between the beneficial microorganisms in the rhizosphere.
\end{abstract}

Key words: frequency mycorrhization percentage, mycorrhization intensity, colonized root biomass.

\section{Introducción}

El trigo (Triticum aestivum L.) es uno de los cultivos más importantes a nivel nacional presentando gran importancia socioeconómica, por ser cultivado mayoritariamente por pequeños productores (Larraín y Olfos, 2012). La superficie total sembrada durante la temporada 2011/2012 cubrió las 245.231 ha, de las cuales la Región de La Araucanía aportó con la mayor superficie alcanzando las 106.791 ha

1 Escuela de Agronomía. Facultad de Recursos Naturales. Universidad Católica de Temuco (UCTemuco). Rudecindo Ortega 02950. Temuco, Chile.

2 Núcleo de Investigación en Producción Alimentaria. UCTemuco.

* Autor para correspondencia: ccastill@uct.cl

Fecha de Recepción: 8 Julio, 2015.

Fecha de Aceptación: 10 Marzo, 2016. 
(Larraín y Olfos, 2013). La productividad triguera nacional se sustenta en el empleo de variedades de alto potencial de rendimiento, que requieren un uso intensivo de insumos como fertilizantes, enmiendas calcáreas y pesticidas (Fundación Chile, 2005). Como consecuencia de la fertilización se afectan los procesos biológicos, que involucran las poblaciones de microorganismos benéficos que habitan la rizósfera de los agroecosistemas causando un deterioro constante de los suelos (López et al., 2007). Como alternativa se están utilizando biofertilizantes y bioestimuladores microbianos, que constituyen un medio ecológicamente aceptable para reducir insumos externos y mejorar la cantidad y calidad de los recursos internos. Así, al utilizar microorganismos seleccionados, se aporta a los cultivos nitrógeno (N) fijado de la atmósfera, fósforo $(\mathrm{P})$ disponible a partir de formas escasamente solubles en el suelo y sustancias fisiológicamente activas que al interactuar con la planta, desencadenan una mayor actividad del metabolismo vegetal (Chaturvedi, 2006).

Entre los microorganismos rizosféricos benéficos se encuentran los hongos micorrícicos arbusculares (HMA), que constituyen una de las asociaciones simbióticas mutualísticas más importantes de la naturaleza. La asociación se establece entre las raíces de aproximadamente el $80 \%$ de las plantas terrestres y hongos pertenecientes al phylum Glomeromycota, que tienen una amplia distribución en el planeta (Smith y Read, 2008). Los HMA actúan como un complemento de la raíz en la captación de nutrientes, especialmente P y N (Colozzi Filho y Cardoso, 2000), debido al micelio extrarradical que permite la exploración de un mayor volumen de suelo (Clark y Zeto, 2000). Sin embargo, no todas las asociaciones entre HMA-planta son compatibles, pudiendo beneficiarse en mayor grado un hospedero $\mathrm{y}$ a su vez el hongo adaptarse a distintas condiciones edafoclimáticas, evidenciando marcadas diferencias, no sólo estructurales, sino también funcionales entre especies, e incluso morfotipos de una misma especie (Linderman y Davis, 2004). Así, Gavito y Miller (1998) informaron una disminución en la colonización fúngica de un cultivo hospedero, al incluir en la rotación un cultivo cabecera no hospedero, en comparación con rotaciones que incluyen plantas antecesoras micotróficas (Castillo et al., 2006). Al considerar la importancia que tiene la colonización HMA temprana en la absorción de $\mathrm{P}$, esta disminución en la micorrización producida por un precultivo no hospedero, podría afectar la producción del cultivo siguiente, a través de cambios en el potencial de inóculo del suelo y la consecuente colonización (Gavito y Miller, 1998; Castillo et al., 2008).

Por otra parte, los HMA pueden estimularse por otros microorganismos o productos naturales presentes en la rizósfera, acelerando la colonización de las raíces (Siqueira et al., 2002). Los flavonoides como formononetina estimulan la colonización fúngica (Siqueira et al., 1991) y bacterias de vida libre, pueden liberar iones fosfato que captan las hifas HMA para la planta hospedera (Bonfante y Anca, 2009). Estos mecanismos alternativos que ocurren en la rizósfera pueden estimular el desarrollo radicular, causando en las raíces una mayor susceptibilidad a la colonización HMA y mejorando el reconocimiento entre la raíz y los hongos (Sarabia et al., 2010).

De acuerdo a lo anterior, el objetivo de este trabajo fue estudiar en una rotación raps-trigo establecida en campo en un Andisol de la Región de La Araucanía, el efecto de la aplicación de tres productos naturales comerciales (Myconate, Fosfobio y Natural Green) sobre la micorrización temprana por HMA.

\section{Materiales y Métodos}

En julio de 2012 se estableció el ensayo en la localidad de Vilcún, Región de La Araucanía (38³9' lat. Sur; $72^{\circ} 03^{\prime}$ long. Oeste); el año anterior el terreno fue sembrado con raps, un no hospedero micorrícico. La cosecha final se realizó en febrero de 2013, a los 111 días después de la siembra (DDS). Durante el desarrollo del ensayo, con la finalidad de cuantificar la colonización HMA temprana del trigo se realizaron tres muestreos de plantas, a los 44 DDS (Z 1.2), 63 DDS (Z 2.2) y 77 DDS (Z 2.3) (Zadoks, 1974).

El cereal utilizado fue trigo variedad CracBaer en una dosis de $200 \mathrm{~kg} \mathrm{ha}^{-1}$. La fertilización se realizó en forma parcializada, con una primera aplicación (a la siembra) de $333 \mathrm{~kg}$ de mezcla 7:27:8:6(S):14(Ca) ha $^{-1}$ y otras dos aplicaciones cada 2 meses, con salitre potásico (equivalente a $50 \mathrm{~kg} \mathrm{~N} \mathrm{ha}^{-1}$ ).

El diseño experimental correspondió a un cuadrado latino con cuatro repeticiones y cuatro tratamientos: a) un control (C) sin aplicación de producto natural (PN); b) Fosfobio (F) $(100 \mathrm{~mL}$ $\left.\mathrm{ha}^{-1}\right)$, PN que contiene bacterias solubilizadoras 
de fosfato (Azotobacter sp., Bacillus megaterium de Bary y Bacillus polymyxa); c) Myconate (M) $\left(100 \mathrm{~g} \mathrm{ha}^{-1}\right)$, PN que tiene como ingrediente activo formononetina, y d) Natural Green $(\mathrm{N})(2,5 \mathrm{~kg}$ $\mathrm{ha}^{-1}$ ), PN que contiene principalmente carbonato de calcio. Las unidades experimentales fueron 16, presentando cada parcela una dimensión de $4 \times 3 \mathrm{~m}$.

La colonización HMA en las raíces del trigo se cuantificó por el número de puntos de entrada (PE), el porcentaje de frecuencia de micorrización $(\% \mathrm{~F})$ y la intensidad de micorrización $(\% \mathrm{M})$ según metodología propuesta por Trouvelot et al. (1986) y la biomasa de raíz colonizada (BRC) de acuerdo con Castillo et al. (2008).

Desde cada parcela experimental, a los 44 DDS, 63 DDS y 77 DDS, se cosecharon al azar cinco plantas de trigo. En cada una se cortó la parte aérea y se separaron las raíces que se lavaron con abundante agua y se tiñeron con una solución de azul de tripán 0,05\% p/v (Phillips y Hayman, 1970). Para cuantificar los parámetros de micorrización HMA, se colocaron en un portaobjeto 10 trozos de raíces, que se observaron bajo un microscopio contabilizando el número de $\mathrm{PE}$, de acuerdo con las hifas que formaron un apresorio sobre la corteza y el $\% \mathrm{~F}$ y $\% \mathrm{M}$, se determinó por la presencia de estructuras fúngicas usando categorías de 1 a 5 (Trouvelot et al., 1986).

\section{Análisis estadístico}

Para cada parámetro se verificó normalidad y homogeneidad, seguido de un análisis de varianza utilizando ANDEVA de una vía y el test de comparación múltiple de Tukey $(\mathrm{p} \leq 0,05)$. Los valores de frecuencia e intensidad fueron transformados por el arco seno de la raíz cuadrada del porcentaje para normalizar su distribución. Para el análisis estadístico de los datos se utilizó el software SPSS para Windows versión 20.0.

\section{Resultados y Discusión}

A los 44 DDS, el mayor número de PE de los HMA en las raíces de trigo se obtuvo con el tratamiento adicionado con Myconate, presentando diferencias significativas en relación a los otros dos $\mathrm{PN}$ y con el control. Estos resultados muestran el efecto beneficioso de Myconate en los primeros estadios de desarrollo del cultivo. La adición de Natural Green también aumentó significativamente los PE en comparación con el control, pero en menor magnitud que Myconate (Figura 1). A los 63 DDS no se observaron diferencias entre los distintos tratamientos y tardíamente a los 77 DDS, en el control aumentaron significativamente los PE con respecto a los otros tratamientos adicionados con PN, donde la micorrización estaba más avanzada. En relación con la formononetina, principal componente de Myconate, Siqueira et al. (1991) informaron que actúa como inductor en la formación de apresorios, aumentando la densidad de PE de los HMA en la raíz y facilitando la interacción hongo-planta, lo que influye en el desarrollo de las micorrizas.

A los 44 DDS, en la frecuencia de micorrización $(\% \mathrm{~F})$ de las raíces del trigo se observaron diferencias entre Myconate sobre Fosfobio (Figura 2) mientras que, a los 77 DDS, significativas frecuencias de micorrización se alcanzaron con Fosfobio y el control. Los resultados muestran que la formononetina estimula la colonización HMA temprana durante las primeras etapas de desarrollo del cultivo. Por el contrario, Fosfobio estimuló la frecuencia de micorrización en el último muestreo, a los 77 DDS. Según Linderman (1997), las rizobacterias tendrían un efecto estimulante sobre el crecimiento de HMA, lo que concuerda con lo informado por Sarabia et al. (2010) sobre la estimulación que ejercerían los microorganismos en el desarrollo radicular, es decir, una mayor susceptibilidad de la raíz a la colonización por HMA y la mejora del proceso de reconocimiento entre la raíz y los hongos.

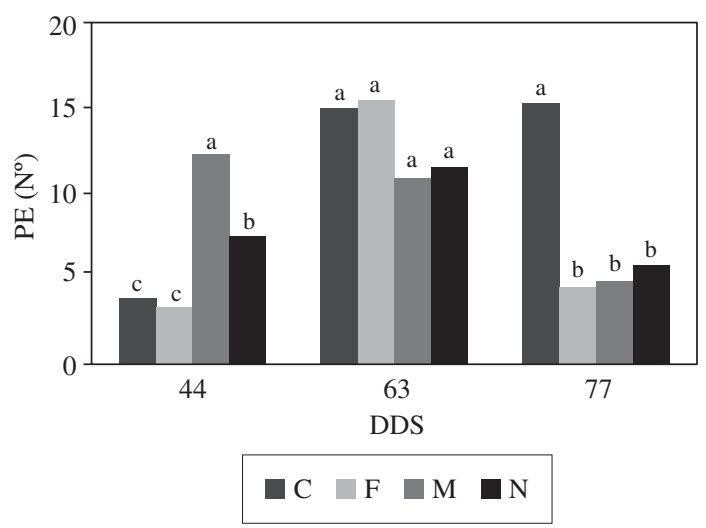

Figura 1. Puntos de entrada de hongos micorrícicos arbusculares en raíces de T. aestivum variedad Crac-Baer, a los 44 DDS, 63 DDS y 77 DDS, con aplicación de tres PN comerciales ( $\mathrm{C}=$ Control, $\mathrm{F}=$ Fosfobio, $\mathrm{M}=$ Myconate, $\mathrm{N}=$ Natural Green $)$. En cada tiempo, barras seguidas con distinta letra indican diferencias significativas según test de Tukey $(\mathrm{p} \leq 0,05)$. 


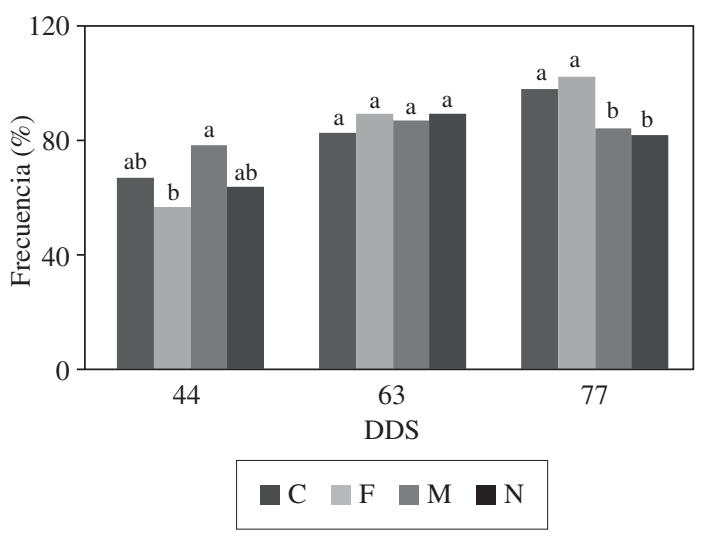

Figura 2. Frecuencia de micorrización HMA en raíces de $T$. aestivum variedad Crac-Baer, a los 44 DDS, 63 DDS y 77 DDS, con aplicación de tres $\mathrm{PN}$ comerciales $(\mathrm{C}=\mathrm{Control}$, $\mathrm{F}=$ Fosfobio, $\mathrm{M}=$ Myconate, $\mathrm{N}=$ Natural Green). En cada tiempo, barras seguidas con distinta letra indican diferencias significativas según test de Tukey $(\mathrm{p} \leq 0,05)$.

En relación con la intensidad de colonización (\%M) en la raíces de trigo (Figura 3) a los 63 DDS, se encontraron diferencias significativas entre Natural Green sobre Fosfobio. A los 77 DDS, la intensidad de colonización con Fosfobio incrementó en un 58\% sobre el tratamiento adicionado con Myconate y en un 57\% sobre Natural Green. Estudios realizados por McGonigle y Miller (1993) encontraron que la colonización HMA del trigo sigue una curva sigmoidal, comenzando con una fase de latencia corta que fluctúa entre los 15 DDS y 19 DDS, seguida por un crecimiento de la colonización

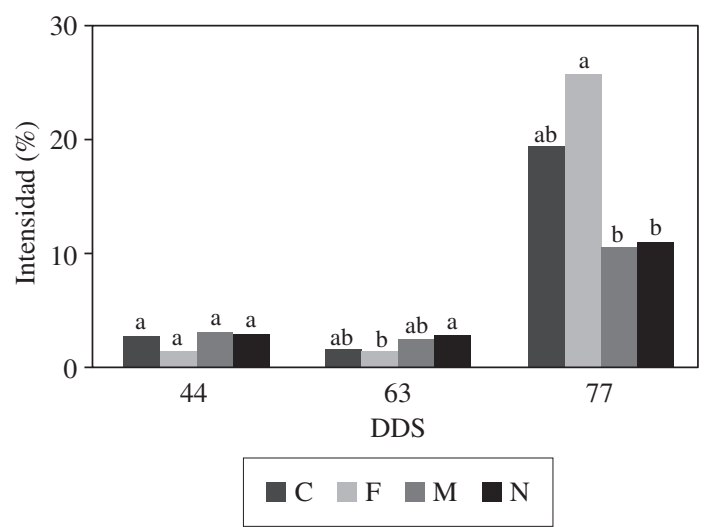

Figura 3. Intensidad de colonización HMA en T. aestivum variedad Crac-Baer, a los 44 DDS, 63 DDS y 77 DDS con aplicación de tres $\mathrm{PN}$ comerciales $(\mathrm{C}=$ control, $\mathrm{F}=$ Fosfobio, $\mathrm{M}=$ Myconate, $\mathrm{N}=$ Natural Green). En cada tiempo, barras seguidas con distinta letra indican diferencias significativas según test de Tukey $(\mathrm{p} \leq 0,05)$. que alcanza su máximo a los 50 DDS, para luego disminuir linealmente. Una tendencia similar a la observada en los dos primeros muestreos.

A los 44 DDS, la BRC en el trigo, presentó diferencias entre el tratamiento con Natural Green sobre Fosfobio (Figura 4). A los 77 DDS fue significativamente mayor en el control y el tratamiento con Fosfobio. En general, a través del tiempo, se observa una curva ascendente en la BRC, resultados que concuerdan con los informados por Castillo et al. (2012) trabajando con distintos hospederos que incluyeron al T. aestivum, con notable aumento de la biomasa a los 30 DDS y 45 DDS.

A los 44 DDS, con el tratamiento adicionado de Fosfobio, se obtuvieron las plantas de T. aestivum var. Crac-Baer de mayor altura (Figura 5). A los 63 DDS, entre PN, Myconate mostró un incremento de $16 \%$, en comparación con Natural Green, mientras a los 77 DDS se observaron diferencias de $21 \%$ en altura entre las plantas tratadas con Fosfobio y el control. Según Sarabia et al. (2010) la simbiosis micorrícica facilita la captación de P, influyendo también directa o indirectamente en la absorción de otros iones minerales que promueven un mayor crecimiento de las plantas especialmente en suelos de baja disponibilidad.

En relación a la longitud radical no se observaron diferencias significativas entre PN con el control. Según Berta et al. (1993) uno de los efectos beneficiosos de los HMA consiste en incrementar la proporción de raíces secundarias con respecto a las primarias; así, en un sistema de raíces fino y

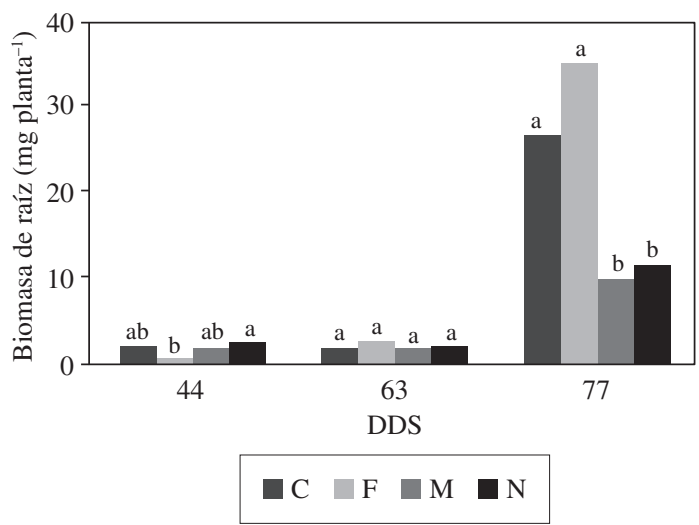

Figura 4. Biomasa de raíz colonizada en T. aestivum variedad Crac-Baer, a los 44 DDS, 63 DDS y 77 DDS, con aplicación de tres $\mathrm{PN}$ comerciales $(\mathrm{C}=\mathrm{Control}, \mathrm{F}=$ Fosfobio, $\mathrm{M}=$ Myconate, $\mathrm{N}=$ Natural Green). En cada tiempo, barras con distinta letra indican diferencias significativas según test de Tukey $(\mathrm{p} \leq 0,05)$. 


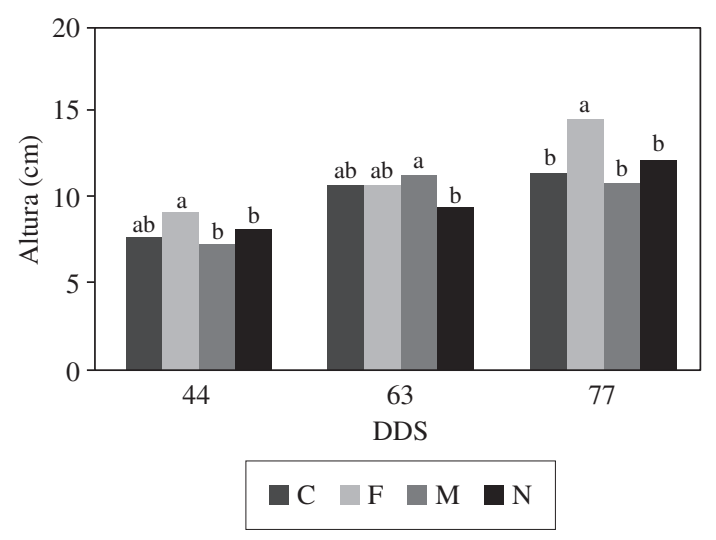

Figura 5. Altura de planta en T. aestivum variedad Crac-Baer, a los 44 DDS, 63 DDS y 77 DDS, con aplicación de tres $\mathrm{PN}$ comerciales $(\mathrm{C}=$ Control, $\mathrm{F}=$ Fosfobio, $\mathrm{M}=$ Myconate, $\mathrm{N}=$ Natural Green). En cada tiempo, barras seguidas de distinta letra indican diferencias significativas según test de Tukey $(\mathrm{p} \leq 0,05)$.

elongado la absorción de nutrientes resulta ser más efectiva (McCully, 1999). Al igual que en la longitud radical, también en el peso seco aéreo (PSA) no se observaron diferencias entre los tratamientos adicionados con $\mathrm{PN}$ y el control.

En relación al peso seco de la raíz (PSR), a los 63 DDS, el tratamiento con Myconate presentó un peso significativamente mayor que Natural Green y el control mientras que, a los 77 DDS, el mayor peso de raíz correspondió al control (Figura 6). En estudios realizados con 10 cultivares de trigo, Borie et al. (1998) observaron que los HMA resultaron beneficiosos para la formación de materia seca.

En este trabajo en campo, el potencial fúngico podría encontrarse exacerbado por la presencia de malezas hospederas que acompañaron al cultivo (Castillo et al., 2008). Por otra parte, el efecto que tendrían los PN en la micorrización temprana del trigo al usar como precultivo un no hospedero, establecido en un suelo típico de la región, resultan ser un estudio pionero en el tema de la sustentabilidad de los agroecosistemas que ayudan a dilucidar las relaciones que se establecen entre los microorganismos benéficos de la rizósfera. Sin embargo, surgen algunas interrogantes, como lo que sucedería en la micorrización temprana del trigo,

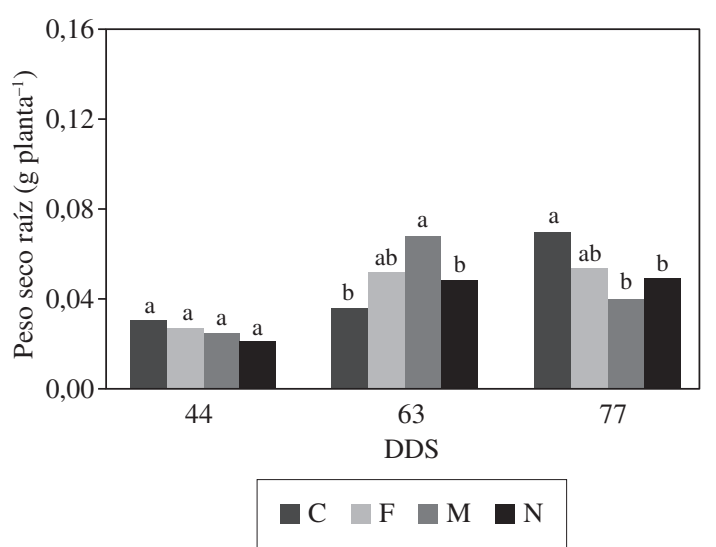

Figura 6. Peso seco radical de T. aestivum variedad Crac-Baer, a los 44 DDS, 63 DDS y 77 DDS, con aplicación de tres $\mathrm{PN}$ comerciales $(\mathrm{C}=$ Control, $\mathrm{F}=$ Fosfobio, $\mathrm{M}=$ Myconate, $\mathrm{N}=$ Natural Green). En cada tiempo, barras seguidas de distinta letra indican diferencias significativas según test de Tukey $(\mathrm{p} \leq 0,05)$.

al usar otros PN o rotaciones de cultivos sólo con hospederos micorrícicos.

\section{Conclusiones}

A los 44 DDS, en la rotación en campo rapstrigo, la aplicación de Myconate a la semilla de trigo variedad Crac-Baer, benefició la colonización temprana de hongos micorrícicos arbusculares, medida por un mayor número de puntos de entrada y frecuencia de micorrización, en relación con el control.

Con la adición de PN al cultivo de trigo se obtuvieron en las raíces distintas intensidades de colonización. La mayor intensidad se alcanzó con Natural Green a los 63 DDS y con Fosfobio a los 77 DDS.

A los 77 DDS, con la aplicación de Fosfobio al trigo, la biomasa de raíz colonizada aumentó significativamente en relación con los otros tratamientos.

\section{Agradecimientos}

Los autores agradecen al Proyecto FONDECYT $\mathrm{N}^{\mathrm{o}} 11090014$, por el financiamiento otorgado para la ejecución de la investigación. 


\section{Literatura Citada}

Berta, G.; Fusconi, A.; Trotta, A.

1993. VA mycorrhizal infection and the morphology and function of root systems. Environmental and Experimental Botany, 33 (1): 159-173.

Bonfante, P.; Anca, I.

2009. Plants, mycorrhizal fungi, and bacteria: a network of interactions. Annual Review Microbiology, 63: 363-383.

Borie, F.; Rubio, R.; Schälchli, C.

1998. Micorrizas arbusculares y actividad fosfatásica de diez cultivares de trigo. Agricultura Técnica, 58: 47-55.

Castillo, C.G.; Puccio, F.; Morales, D.; Borie, F.; Sieverding, E. 2012. Early arbuscular mycorrhiza colonization of wheat, barley and oats in Andosols of Southern Chile. Journal of Soil Science and Plant Nutrition, 12 (3): 511-524.

Castillo, C.G.; Rubio, R.; Rouanet, J.L.; Borie, F.

2006. Early effects of tillage and crop rotation on arbuscular mycorrhizal fungal propagules in an Ultisol. Biology Fertility of Soils, 43: 83-92.

Castillo, C.G.; Astroza, I.; Borie, F.; Rubio, R.

2008. Efecto de cultivos hospederos y no hospederos sobre propágulos micorrícicos arbusculares. Journal of Soil Science and Plant Nutrition, 12 (3): 511-524.

Chaturvedi, I.

2006. Effects of phosphorus levels alone or in combination with phosphate-solubilizing bacteria and farmyard manure on growth, yield and nutrient up-take of wheat (Triticum aestivum L.). Journal of Agriculture and Social Sciences, 2 (2): 96-100.

Clark, R.B.; Zeto, S.K.

2000. Mineral acquisition by arbuscular mycorrhizal plants. Journal Plant Nutrition, 23: 867-902.

Colozzi Filho, A.; Cardoso, E.J.

2000. Detecção de fungos micorrizicos arbusculares em raízes de cafeeiro e de crotolária cultivada na entrelinha. Pesquisa Agropecuária Brasileira, 35 (10): 2033-2042.

Fundación Chile

2005. Una nueva visión para el sector triguero en Chile. Fundación Chile. Santiago, Chile, 100 p.

Gavito, M.E.; Miller, M.H.

1998. Changes in mycorrhiza development in maize induced by crop management practices. Plant and Soil, 198: 185-192.

Larraín, J.D.; Olfos G., M.J.

2012. Boletín de trigo: Producción, precios y comercio exterior. Enero-febrero 2012. Odepa. Santiago Chile. 44 p. Disponible en http://www.odepa.gob.cl//odepaweb/ servicios-informacion/Boletines/BTrigo_enefeb12.pdf;j sessionid=AFEA695BCF4299B09D734134CF21EEC1. Consultado: 23/Dic/2014.

Larraín, J.D.; Olfos G., M.J.

2013. Boletín de trigo: Producción, precios y comercio exterior. Diciembre 12 - enero 2013. Odepa. Santiago Chile. 34 p. Disponible en http://www.odepa.gob.cl//odepaweb/
servicios-informacion/Boletines/boletinTrigoEnero2013. pdf;jsessionid=520A7424A0179B686496D917B97AD4 8C. Consultado: 23/Dic/2014.

Linderman, R.G.

1997. Vesicular-arbuscular mycorrhizal (VAM) fungi. In: Caroll, G.C.; Tudzynski, P. (eds.). The Mycota. Berlin, Germany: Springer-Verlag., pp. 117-128.

Linderman, R.G.; Davis, E.A.

2004. Varied response of marigold (Tagetes spp.) genotypes to inoculation with different arbuscular mycorrhizal fungi. Scientia Horticulturae 99: 67-78.

López, M.; López de Rojas, I.; España, M.; Izquierdo, A.; Herrera, L.

2007. Efecto de la fertilización inorgánica sobre la disponibilidad de nutrimentos en el suelo, nivel nutricional de la planta y hongos micorrízicos arbusculares en plantaciones de Theobroma cacao L. Agronomía Tropical 57 (1): 31-43.

McCully, M.

1999. Roots in soil: unearthing the complexities of roots and their rhizosphere. Annual Review of Plant Physiology and Plant Molecular Biology 50: 695-718.

McGonigle, T.P.; Miller, M.

1993. Mycorrhizal development and phosphorus absorption in maize under conventional and reduced tillage. Soil Science Society of American Journal 57 (4): 1002-1006.

Phillips, J.; Hayman, D.

1970. Improved procedures for cleaning roots and staining parasitic and vesicular arbuscular mycorrhizal fungi for rapid assessment of infection. Transanctions of the British Mycologycal Society, 55: 158-161.

Sarabia, M.; Madrigal, R.; Martínez, M.; Carreón, Y.

2010. Plantas, hongos micorrícicos y bacterias: su compleja red de interacciones. Biológicas, 12 (1): 65-71.

Siqueira, J.O.; Nair, M.G.; Hammerschimidt, R.; Safir, G.R.

1991. Significance of phenolic compounds in plant-soilmicrobial systems. Critical Review in Plant Science, 10 (1): 63-121.

Siqueira, J.O.; Lambais, M.R.; Sturmer, S.L.

2002. Fungos micorrízicos arbusculares: características, associação simbiótica e aplicação na agricultura. Biotecnologia Ciência e Desenvolvimento, 25: 12-21.

Smith, S.E.; Read, D.J.

2008. The mycorrhizal symbiosis. Londres, R.U.: Academic Press, $3^{\mathrm{a}}$ ed., $787 \mathrm{p}$.

Trouvelot, A.; Knough, J.L.; Gianinazzi-Pearson, V.

1986. Mesure du taux de mycorrhization VA d'un systeme radiculaire. In: Gianinazzi-Pearson, V.; Gianinazzi, S. (eds.). Physiological and genetical aspects of mycorrhizae. Paris, Francia: INRA Press, pp. 217-221.

Zadoks, J.C.; Chang, T.T.; Konzak, C.F.

1974. A decimal code for the growth stages of cereals. Weed Research 14: 415-421. 